\title{
Harnessing Vibration Energy from a Piezoelectric Cantilever Beam through a Waist-High Tripod Turnstile and Magnetic Flywheel
}

\author{
Esperanza E. Chua ${ }^{1+}$, Glenn V. Magwili ${ }^{2}$, Phil Harold O. Gealan ${ }^{3}$, John Benedict T. Dimero ${ }^{4}$, \\ Jorel Luis B. Fernando ${ }^{5}$ and Nars-Icon Z. Tarun ${ }^{6}$ \\ ${ }^{1}$ School of Electrical, Electronic and Computer Engineering, Mapua University, Manila, Philippines
}

\begin{abstract}
Searching for energy sources that poses less environmental impact and can be sustained for a certain time leads to renewable energy. Many people are familiar with the usage of ambient renewable energy source, including solar and wind, hence, taking for granted wasted energy sources, such as vibrations. The idea of harvesting energy from any type of vibration, whether it is physically created, mechanically generated, or electromagnetically produced, is now possible due to the discovery of piezoelectric effect. The aim of this study was to harness vibration energy from a piezoelectric cantilever beam through a waist-high tripod turnstile and magnetic flywheel. The prolongation of magnetic flywheel that harnessed and stored electrical energy was also investigated. In this study, the prolongation of rotation of the magnetic flywheel was achievable by multiple actuations in the waist-high tripod turnstile. A maximum increase of storage capacity of $4.455 \%$ from an initial storage of $2.563 \%$ was harnessed and stored from the setup. The harnessed and stored electrical energy accounted for supplementary source of energy for other primary sources of electrical energy.
\end{abstract}

Keywords: Turnstile, Magnetic Flywheel, Piezoelectric, Vibration, Renewable Energy, Harnessing Energy

\section{Introduction}

In an international energy outlook of 2016, the economic growth, along with accompanying structural changes involving living standards, strongly influence world energy consumption, leading to rapid growth of energy demand. Production of today's renewable energy sources still is not enough, as compared to what oil brings. With constant innovation, the researchers are putting much attention to increase efficiency of harnessing energy from vibrations. The discovery of piezoelectric effect motivates the idea of harvesting energy from the said source.

To reduce further depletion of non-renewable energy sources, like oil and coal, the awareness on the need for the use of renewable energy has increased. This awareness inspired the researchers to look into the application of piezoelectric technology to harness vibration energy and use it to produce electrical energy.

This research aims to harness the vibration energy from a piezoelectric cantilever beam through a waisthigh tripod turnstile and magnetic flywheel. The researchers built a waist-high tripod turnstile with piezoelectric cantilever beam and magnetic flywheel using permanent magnets, along with a charging circuit. The length of time that the rotation of the flywheel can be prolonged in single/multiple actuations was determined. Also, the amount of electrical energy that can be harnessed and stored from the setup was determined. The result of this study helps to promote more ways of harvesting renewable energy from sources such as human effort.

\footnotetext{
+ Corresponding author. Tel.: + 639232097574

E-mail address: gvmagwili@mapua.edu.ph, eechua@mapua.edu.ph.
} 


\section{Methodology}

The researchers collected vibration energy in waist-high tripod turnstile from its interaction with the magnetic flywheel and permanent magnets. This was simulated at the entrance of campus of Mapua University from November 5-11, 2017 and the parameters measured were stored at data logger. The harvested energy was stored in an 18350 Lithium Ion Rechargeable Battery, with 3.7VDC, $650 \mathrm{mAh}$.

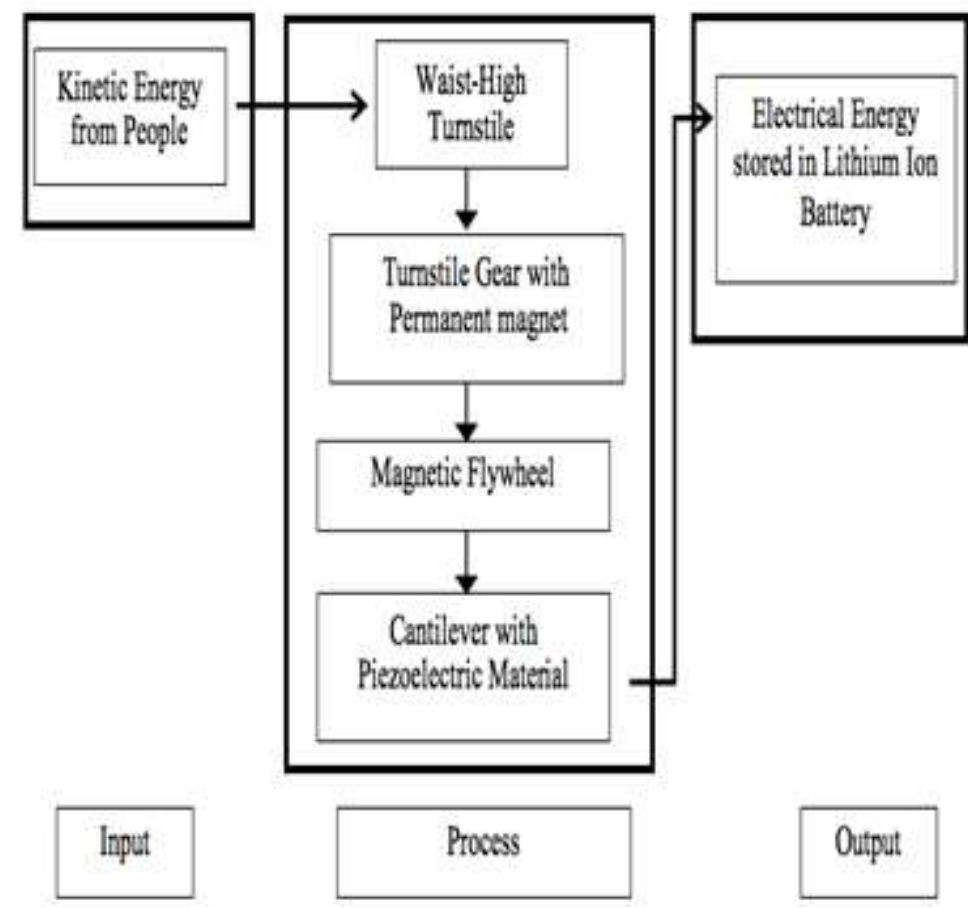

Fig. 1: Conceptual Framework of the Energy Harvesting System.

Figure 1 shows that the kinetic energy from human effort in turning the waist-high turnstile will cause the directed magnetic flywheel to rotate with prolonged motion. It is due to the shaft of turnstile directed to the magnetic flywheel. The rotation of the magnetic flywheel caused vibrations in the piezoelectric cantilever beam due to influence of magnetism of permanent magnets beside the beam. This will stress the piezoelectric material in the cantilever and will start generating energy[1]. The harnessed energy would be stored in a battery[2], which can be used for powering up low-powered devices.

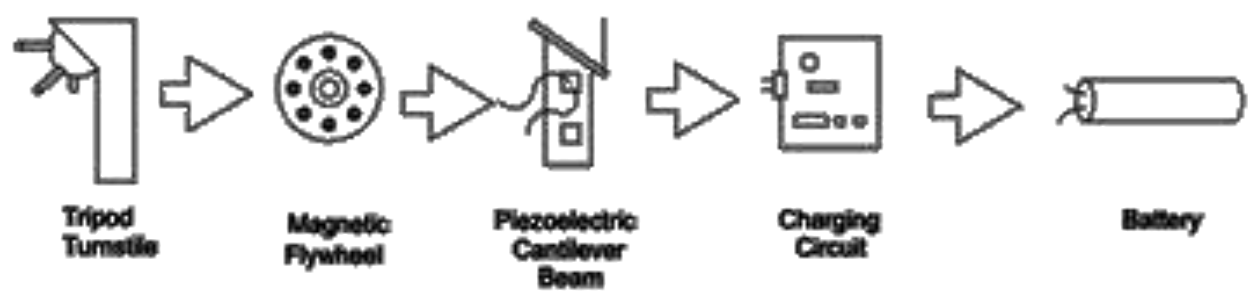

Fig. 2: Connection Flow of the System.

Figure 2 shows the basic connection flow of the system. It also shows the hardware and circuit components that will be connected and used in the system. Subsequent to this, Figure 3 shows the design layout of the waist-high tripod turnstile in three-dimension. 


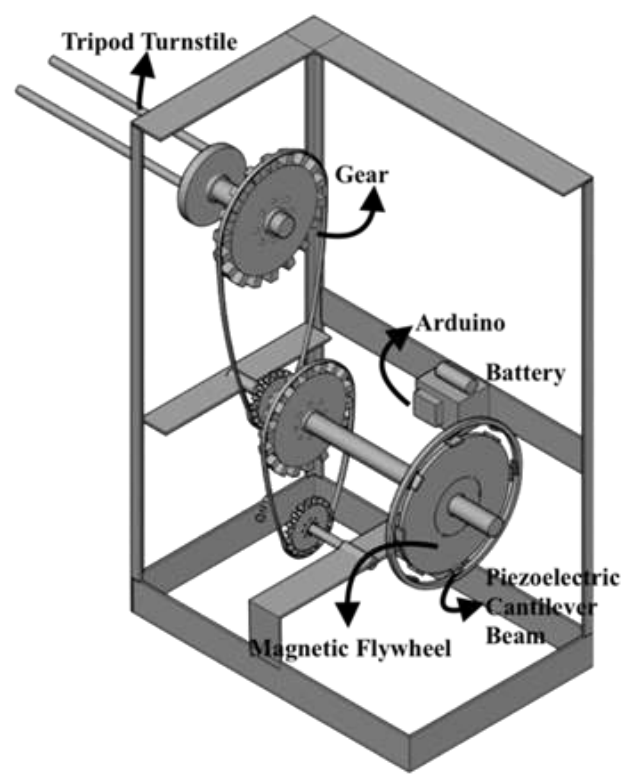

Fig. 3: Design Layout of the System (3D).
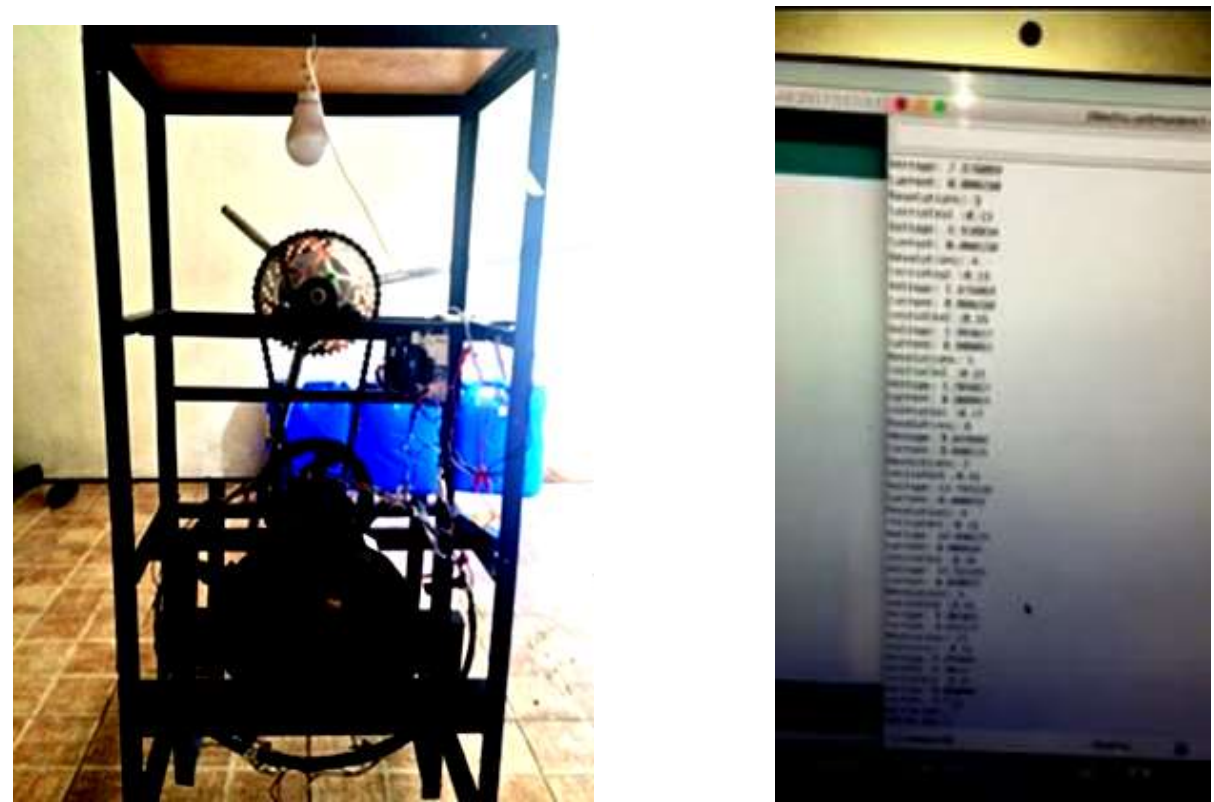

Fig. 4: Hardware Setup of Waist-High Tripod Turnstile and Software Setup for Monitoring of Parameters.

Figures 4 shows the actual setup, in terms of hardware and software. The software setup was based on the IEEE Std. 1855, entitled "Arduino ${ }^{\mathrm{TM}}$-based fuzzy Systems", while the parameters that was measured was based on IEEE Std. 177, entitled "Definitions and Methods of Measurement for Piezoelectric Vibrators), with the storing of electrical energy was based on IEEE Std. 1881, entitled "Glossary of Stationary Battery Terminology."

\section{Results and Discussion}

To determine length of time that the rotation of the flywheel can be prolonged in single/multiple actuations, the prolongation was calculated based on equation (1).

Prolongation $(\mathrm{sec})=$ Time Recorded $-($ Time Basis for Single Actuation + Time Interval $)$

The time basis for single actuation is the highest time recorded when one passerby passes through the turnstile. Table 1 shows that the time basis for single actuation is 7.78 seconds. 
Table 1: Number of Revolutions and Time duration.

\begin{tabular}{|c|c|c|}
\hline Trial & Number of Revolutions & Time (s) \\
\hline 1 & 16 & 6.71 \\
\hline 2 & 16 & 6.46 \\
\hline 3 & 16 & 5.83 \\
\hline 4 & 16 & 6.71 \\
\hline 5 & 14 & 6.36 \\
\hline 6 & 13 & 6.65 \\
\hline 7 & 13 & 6.03 \\
\hline 8 & 11 & 7.25 \\
\hline 9 & 14 & 7.78 \\
\hline 10 & 11 & 6.03 \\
\hline
\end{tabular}

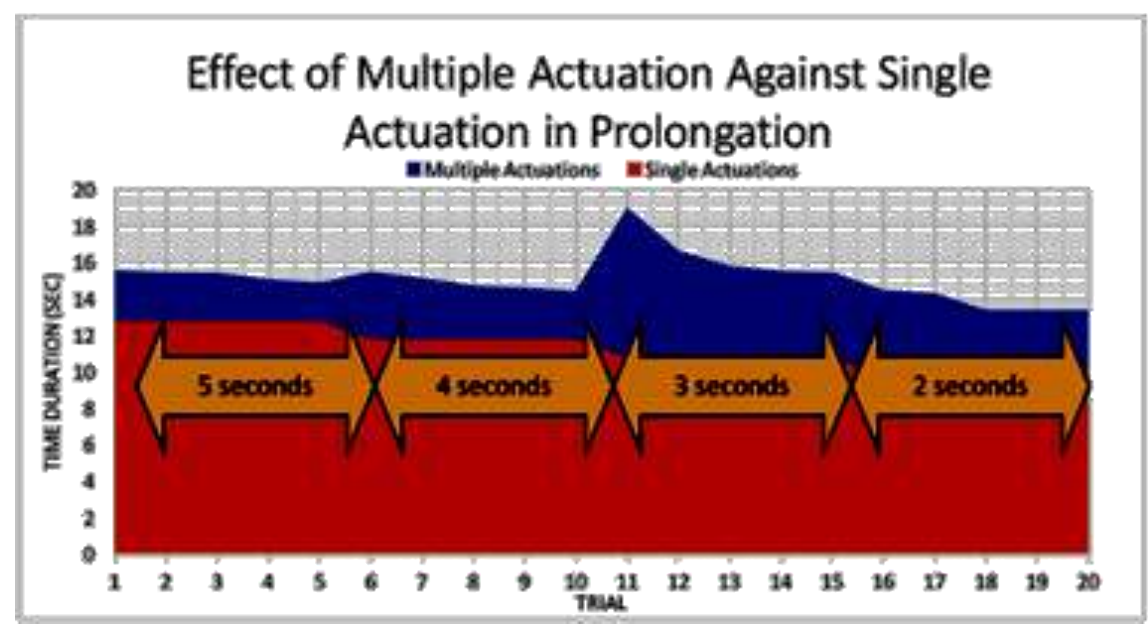

Fig. 5: Effect of Multiple Actuation against Single Actuation in Prolongation.

Figure 5 shows that at 3 seconds time interval obtained the highest prolongation followed by 2 seconds, while 5 seconds time interval has the minimum prolongation when compared to single actuation.

To determine the effect of prolongation in the harnessed energy, the researchers compared the generated voltage by the piezoelectric cantilever at different time intervals against the single actuation during no-load and loaded conditions.

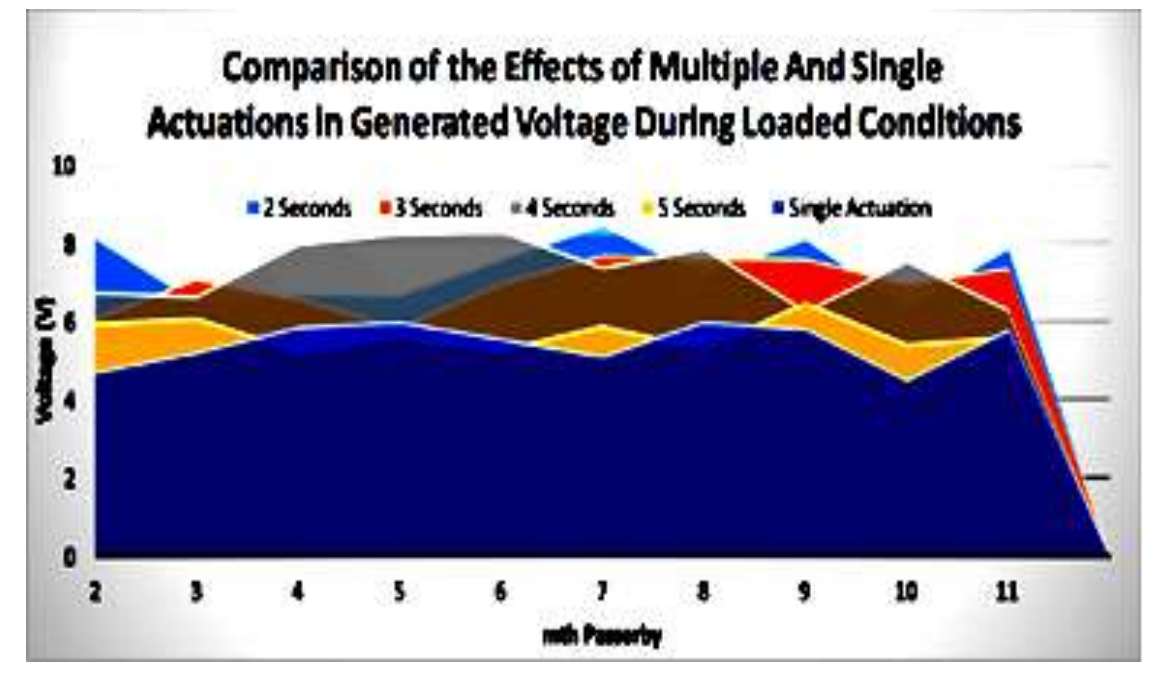




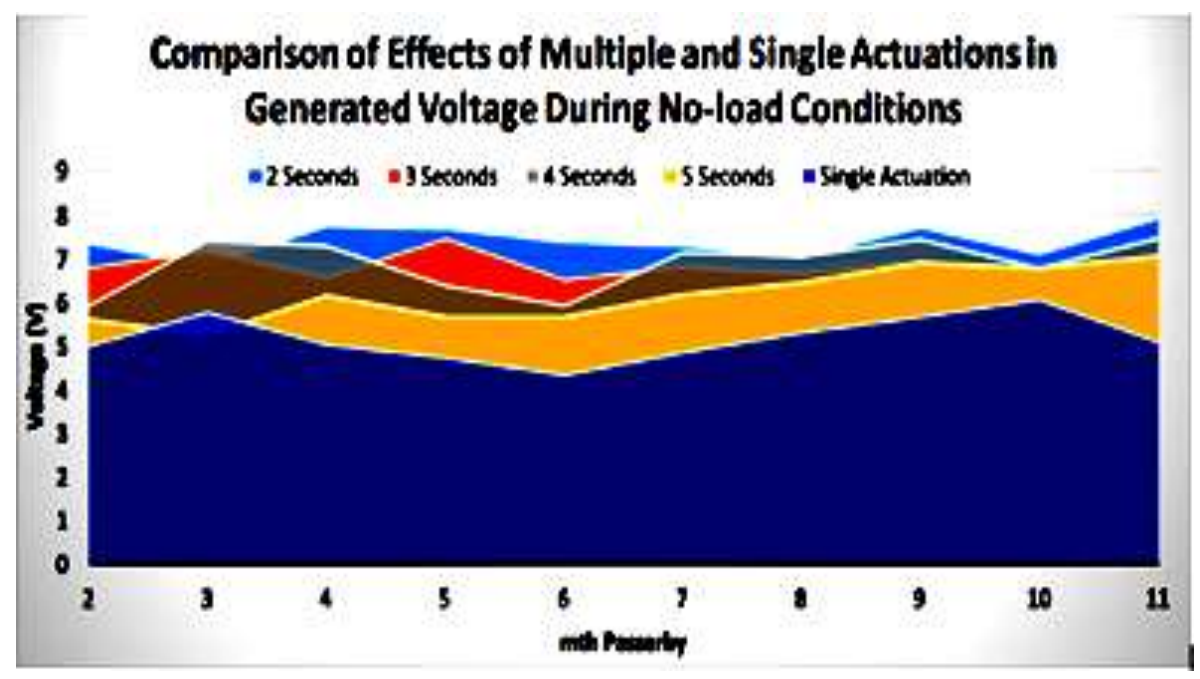

Fig. 6: Comparison of Multiple and Single Actuations in Generated Voltage during No-load and Loaded Conditions

Figure 6 shows that 2 seconds of time interval generated the highest voltage, and can be concluded that the prolongation by the flywheel resulted into additional generated voltage.

The off-peak and peak periods of time, which was based on the number of passersby, were simulated to determine the total harnessed energy stored.
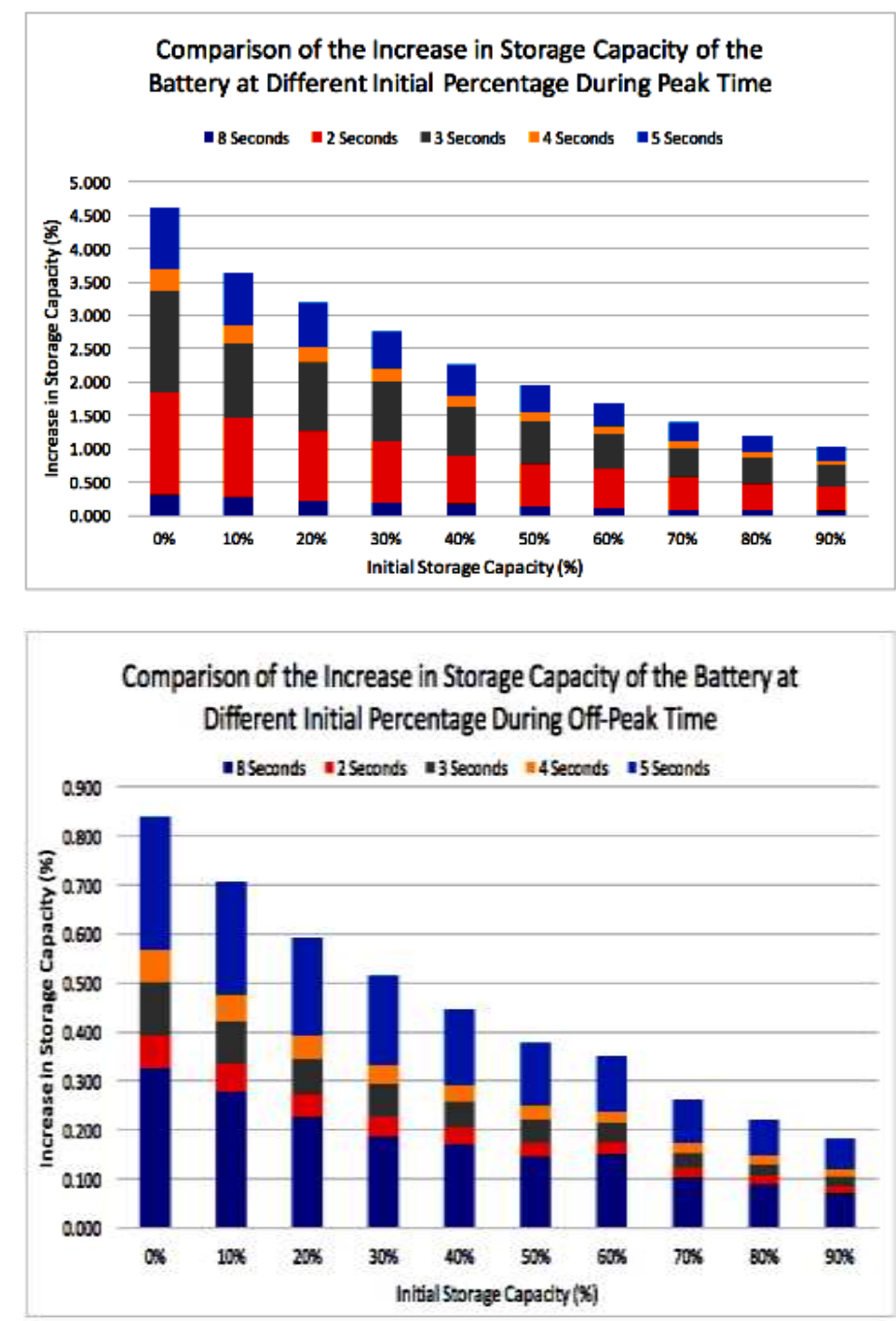

Fig. 7: Comparison of the Increase in Storage Capacity of the Battery at Different Initial Percentage During Peak and Off-Peak Time 
Figure 7 shows an increase of storage capacity approximately $4.5 \%$ and $0.8 \%$ occurred at lowest initial storage capacity during no-load and loaded conditions, respectively.

\section{Conclusion}

The researchers were able to build and harness vibration energy from a waist-high tripod turnstile with piezoelectric cantilever beam and magnetic flywheel, using permanent magnets, incorporated with a charging circuit connected to a battery. Microcontroller was used for efficient and precise measurement and storage of parametric data such as number of revolutions of the magnetic flywheel, generated voltage, current, and power, and storage capacity.

Multiple actuations can be concluded to have significant effect on the prolongation of the magnetic flywheel from which the prolonged time depends on the time interval of entry of the next passer-by. The highest prolongation was obtained on 3 seconds time interval of entry with a mean prolongation of 5.688 seconds.

In terms of electrical energy harnessed, the 2 seconds time interval of entry generated the highest instantaneous generated voltage compared to other time interval of entry. The total increase in storage capacity is maximized when the rotation of the magnetic flywheel is prolonged. A maximum increase in storage capacity of $4.4547 \%$ from an initial storage capacity of $2.5630 \%$ was harnessed and stored from the setup given with peak time condition.

To fully charge the battery, it needs approximately 34 hours of continuous actuation of the tripod turnstile. Thus, it can be concluded that with this amount of increase storage capacity, the system can be used as a supplementary source of energy.

\section{References}

[1] Gao, S., Zhang, G., Jin, L,., Li, P., and Liu, H. (2016) Characteristics of the piezoelectric energy harvesting from the torsional vibration of thin-walled cantilever beams. doi: 10.1109/MESA.2016.7587122

[2] Brown, D. \& Chvala, W., (2017) Flywheel Energy Storage: An Alternative to Batteries for UPS systems. Retrieved January 11, 2017 Dave, M. R., \& Dave, K. C. Analysis of Boost Converter Using PI Control Algorithms. International Journal of Engineering Trends and Technology Volume3 Issue2. 2012. 